\title{
Multiple Equilibrium Behaviors considering Human Exposure to Vehicular Emissions
}

\author{
Zhanbo Sun $\mathbb{D}^{1,2}$ Yu Tan, ${ }^{1,2}$ Rui Ma $\mathbb{D}^{,},{ }^{3}$ Xia Yang, ${ }^{4}$ and Jie Zhang ${ }^{5}$ \\ ${ }^{1}$ National Engineering Laboratory of Integrated Transportation Big Data Application Technology, Chengdu, China \\ ${ }^{2}$ School of Transportation and Logistics, Southwest Jiaotong University, Chengdu, China \\ ${ }^{3}$ Department of Civil and Environmental Engineering, University of California, Davis, CA, USA \\ ${ }^{4}$ Department of Civil Engineering, SUNY Polytechnic Institute, Utica, NY, USA \\ ${ }^{5}$ School of Information Science and Technology, Southwest Jiaotong University, Chengdu, China
}

Correspondence should be addressed to Zhanbo Sun; zhanbo.sun@home.swjtu.edu.cn

Received 23 May 2018; Revised 8 September 2018; Accepted 4 October 2018; Published 21 October 2018

Academic Editor: Zhi-Chun Li

Copyright (C) 2018 Zhanbo Sun et al. This is an open access article distributed under the Creative Commons Attribution License, which permits unrestricted use, distribution, and reproduction in any medium, provided the original work is properly cited.

\begin{abstract}
Emissions produced by urban transportation activities are harmful to people's health and they also affect people's trip-making decisions. In this paper, we explore the multiple equilibrium behaviors considering human exposure to vehicular emissions. We assume that a portion of transportation users are environmental advocates and their route decisions are based on some composite cost functions comprise of a travel time component and an emission exposure component. We then study the multiple equilibrium behaviors with multiple types of users on a traffic network. The multiple equilibrium problems are further converted into variational inequality (VI) problems and they are solved using a method of successive average- (MSA-) based diagonalization method. Per the specific network setting, we find that as travelers become more concerned about their exposure to vehicular emissions, the system emission exposure, travel time, and the total cost get reduced; i.e., Pareto improving solutions are achieved. By analyzing the multiple equilibrium behaviors, we find that the system gets better if more users become environmental advocates. And the change of a small percentage of users should already lead to a good system improvement.
\end{abstract}

\section{Introduction}

Transportation activities account for about $27 \%$ carbon dioxide, 59\% nitrogen oxide, and 54\% carbon monoxide of the atmospheric emissions [1]. Recently, transportation emissions and their negative impacts have been increasingly noticed. Long time exposure to air pollution can cause shortterm and long-term health problems [2]. According to the US Environmental Protection Agency [3], living close to congested urban location has lifetime cancer risks greater than 100 in million, which is about 10 times higher than the national average. This evidence motivated researchers to develop mechanisms to quantify and decrease traffic emissions [4-7]. In the network modeling field, efforts are spent to model and control the network emissions, usually in an aggregated manner, without paying much attention to the impacts of vehicular emissions on people. Travelers are directly exposed to vehicular emissions. And vehicular emissions, in turn, affect traveler's trip-making decisions. Such interrelationship between trip-making decisions and human exposure to vehicular emissions has not been fully studied.

Modeling travelers' trip making behavior is challenging. Taking route choice behavior as an example. The most wellknown theories are the Wardropian principles, including user equilibrium (UE) and system optimum (SO). UE assumes that users always choose the shortest path to minimize their individual cost so that no one can unilaterally change routes to reduce his/her own cost. SO assumes that all users are altruistic to minimize the total system cost. Researchers have recognized there are other users which are neither fully competitive (UE) nor fully cooperative (SO), e.g., the groups of users that follow the Cournot-Nash (CN) behaviors [812]. $\mathrm{CN}$ users are like drivers in a same transport company, 
who try to minimize their group total costs. And different $\mathrm{CN}$ users are fully competitive. The problems that UE, $\mathrm{CN}$, and sometimes SO players coexist in the network are referred to as the mixed equilibrium or multiple equilibrium problems [12]. The work on this topic mostly considered single model objective such as travel time cost $[8,9,11]$. Zhang et al. [10] established a unified framework that simultaneously considered travel time cost and users' time-irrelevant costs. He et al. [13] formulated tradable credit schemes based on the mixed equilibrium network with consideration of users' transaction cost. Yang et al. [12] investigated the mixed equilibrium behaviors with general common constraints and took traffic emissions as a general common constraint. Little work has been done in the network-modeling field to explicitly consider human's emission exposure in the modeling framework.

To capture human exposure to vehicular emissions, it is necessary to describe emission modeling and exposure in an integrated framework (see [14]). Such a framework should encapsulate three components, including (i) a traffic flow model; (ii) an emission model that interprets the source of emission as a function of traffic flow; and (iii) a model that describes how the produced tailpipe emissions spread away from the source and affect the neighborhood. With respect to (i) and (ii), microscopic tools such as Comprehensive Modal Emissions Model (CMEM; [15, 16]), VT-Micro [17, 18], and Motor Vehicle Emission Simulator (MOVES; [19]) can estimate emissions with fine resolution by breaking down the physical behavior of vehicles into different operating modes (e.g., acceleration, deceleration, idle, and cruising). These models, however, require extensive input data such as vehicle type and trajectory information (see [6]) from component (i) and usually do not have analytical forms. These models may not be suitable for real time networkscale applications (route choice modeling). Alternatively, mesoscopic and macroscopic approaches [4, 20-24] can estimate the source of emissions as a function of a set of traffic variables, which are more appropriate for network traffic analysis. In the field of atmospheric environment, component (iii) is known as emission dispersion modeling. Emission dispersion models include Gaussian Plume model [25], Box model [26], Lagrangian model [27], and others [28], among which Gaussian dispersion model is most widely used due to its simplistic nature. Gaussian model-based software packages such as HIWAY2 and California Line Source Dispersion Model version 4 (CALINE4) have been widely adopted to estimate emission concentration and exposure near freeways and major arterials [29-33].

In this study, an integrated modeling framework is applied to analytically estimate vehicular emissions and concentrations within the traffic network. The proposed method adopts the BPR function and a macroscopic emission model to describe the source of emissions. Gaussian dispersion model is then used to capture the dispersion of emissions from the source. The framework can be used to calculate travelers' exposure to emissions, which is considered as a part of the generalized travel cost. We further assume that a portion of transportation users are environmental advocates and their route choice decisions are based on some composite cost functions comprise of a travel time component and an emission exposure component. We then study the multiple equilibrium behaviors with multiple types of users on a traffic network. The multiple equilibrium problems are further converted into variational inequality (VI) problems and they are solved using a method of successive average- (MSA-) based diagonalization method. The model framework proposed in the paper forms the basis for analyzing the more practically meaningful multiple equilibrium behaviors with considerations of people's perception and reaction to emission exposures.

\section{Methodology}

It assumes that multiple types of players may exist on a multiple-origin and multiple-destination network. The first type of users follows Wardropian user equilibrium, and it is referred to as a common UE player who seeks to minimize the individual travel time cost. The second type of users also follows the UE principle, but it considers both travel time and the emission exposure cost, and this type of users is referred to as an environmental advocacy UE player. The third type of users is assumed to be controlled by $\mathrm{CN}$ player(s) whose objective is to minimize the group cost. The equilibrium behaviors under such user composition are referred to as multiple equilibrium hereafter in this paper. Similar definitions can be found in Harker [8], Yang et al. [9], Yang et al. [34], Zhang et al. [10], and Yang et al. [12]. The multiple equilibrium behaviors are studied in the paper to analyze the effects of some environmental-cost-based routing models (e.g., smartphone apps) that provide routing services considering the emission exposure of the transportation users. The notations used in the paper are similar to the ones used in Yang et al. [12].

Assume that all users have been transformed into equivalent passenger car units. We use $\Omega^{U}$ to represent the feasible set for the common UE player, $\Omega^{E}$ to represent the feasible set of the environmental advocacy UE player, and $\Omega^{k}, \forall k \in K$ to represent the feasible set of each environmental advocacy $\mathrm{CN}$ player.

For the common UE player

$\Omega^{U}=\left\{v^{U} \mid v^{U}\right.$ statisfies the following constraints (1)(3)\}:

$$
\begin{aligned}
\sum_{p \in P_{w}} f_{p}^{U} & =D_{w}^{U} \quad \forall w \in W^{U} \\
v_{a}^{U} & =\sum_{p \in P^{U}} \delta_{a p} f_{p}^{U} \quad \forall a \in A \\
f_{p}^{U} & \geq 0 \quad \forall p \in P^{U}
\end{aligned}
$$

For the environmental advocacy UE player $\Omega^{E}=\left\{v^{E} \mid v^{E}\right.$ statisfies the following constraints (4)(6)\}:

$$
\sum_{p \in P_{w}} f_{p}^{E}=D_{w}^{E} \quad \forall w \in W^{E}
$$




$$
\begin{aligned}
& v_{a}^{E}=\sum_{p \in P^{E}} \delta_{a p} f_{p}^{E} \quad \forall a \in A \\
& f_{p}^{E} \geq 0 \quad \forall p \in P^{E}
\end{aligned}
$$

For the environmental advocacy CN players $\Omega^{k}=\left\{v^{k} \mid v^{k}\right.$ statisfies the following constraints (7)(9) $\}, \forall k \in K$ :

$$
\begin{aligned}
\sum_{p \in P_{w}} f_{p}^{k} & =D_{w}^{k} \quad \forall w \in W^{k} \\
v_{a}^{k} & =\sum_{p \in P^{k}} \delta_{a p} f_{p}^{k} \quad \forall a \in A \\
f_{p}^{k} & \geq 0 \quad \forall p \in P^{k}
\end{aligned}
$$

2.1. Emission Exposure Model. A macroscopic model is applied to estimate $\mathrm{CO}$ emission as a function of travel time, as in (10), where $e_{a}\left(v_{a}\right)$ is the $\mathrm{CO}$ emission in grams per vehicle per hour, $l_{a}$ is the length of link $a$ in kilometers, and $t_{a}\left(v_{a}\right)$ is the link travel time in minutes, which takes the form of BPR function (Bureau of Public Roads 1964), as in (11). Similar emission models have been discussed in Wallace et al. [20] and Yin \& Lawphongpanich [4].

$$
\begin{aligned}
& e_{a}\left(v_{a}\right)=0.2038 t_{a}\left(v_{a}\right) e^{0.7962 l_{a} / t_{a}\left(v_{a}\right)} \\
& t_{a}\left(v_{a}\right)=t_{a}^{0}\left(1+\alpha\left(\frac{v_{a}}{c_{a}}\right)^{\beta}\right)
\end{aligned}
$$

For the ease of emission dispersion modeling, we further convert the $\mathrm{CO}$ emission from grams per vehicle per hour to milligram (mg) per meter per second; see (12). Therefore, link $a$ can be treated as a homogeneous line with a linear source strength of $q_{a}$, in $\mathrm{mg} / \mathrm{s}$.

$$
q_{a}\left(v_{a}\right)=0.2038\left(\frac{v_{a}}{3600 l_{a}}\right) t_{a}\left(v_{a}\right) e^{0.7962 l_{a} / t_{a}\left(v_{a}\right)}
$$

Turner [35] proposed the Gaussian equation for a continuous point source, as (13), which is applied to compute the emission concentration resulted from a point source.

$$
\begin{aligned}
& c(x, y, z)=\frac{q}{2 \pi \sigma_{y} \sigma_{z} u} \exp \left(-\frac{y^{2}}{2 \sigma_{y}^{2}}\right) \\
& \cdot\left[\exp \left(-\frac{\left(z-H_{p}\right)^{2}}{2 \sigma_{z}^{2}}\right)+\exp \left(-\frac{\left(z+H_{p}\right)^{2}}{2 \sigma_{z}^{2}}\right)\right]
\end{aligned}
$$

Here $c(x, y, z)$ is the mean concentration at point $(x, y, z)$, in $\mathrm{mg} / \mathrm{m}^{3} ; x$ is an along-wind coordinate measured in wind direction from the source; $y$ is a cross-wind coordinate measured perpendicular to the wind direction; $z$ is an elevation coordinate measured from the ground; $u$ is the mean wind velocity, in $\mathrm{m} / \mathrm{s} ; q$ is the strength of the point source, in $\mathrm{mg} / \mathrm{s}$; $H_{p}$ is the elevation of plume where emissions are released; $\sigma_{y}$ and $\sigma_{z}$ are the standard deviations of the horizontal and vertical dispersion and are dependent upon the atmospheric stability, among other factors. The parameterization of the Gaussian dispersion parameters is out of the scope of the study. Details can be found in Liu et al. [36]. In this paper, we assume that $\mathrm{CO}$ disperses in the near ground, i.e., $\mathrm{z}=0, H_{p}=$ 0 . Therefore, the previous model can be simplified as equation (14a) for any downwind location. And $\mathrm{c}(x, y, z=0)=0$ for any upwind as in (14b).

$$
\begin{aligned}
& c(x, y, z=0)=\frac{q}{\pi \sigma_{y} \sigma_{z} u} \exp \left(-\frac{y^{2}}{2 \sigma_{y}^{2}}\right), \quad x \geq 0 \\
& \mathrm{c}(x, y, z=0)=0, \quad x<0
\end{aligned}
$$

Based on the point emission concentration, the next step is to extend the point source to a line source. Bensen [29] presented a method to decompose each line source (e.g., link $a$ ) into a number of short elements and every element is an equivalent finite line source (FLS) whose center is the element midpoint. Since the points along the equivalent FLS have the same $\mathrm{x}$-coordinate, the emission concentration $C_{e}(x, y)$ caused by short element (or equivalently, FLS) $e$ can be computed by integrating the continuous point source along the length of the FLS, as equation (15). Note that $y_{e}^{1}$ and $y_{e}^{2}$ represent the y-coordinate distances between the two endpoints of equivalent FLS $e$ and the receptor, which can be easily determined given the length of the short element $\left(l_{e}\right)$ and the angle $\left(\varphi_{e}\right)$ between the element and the wind direction, i.e., $y_{e}^{1}=y-l_{e} \sin \varphi_{e} / 2$ and $y_{e}^{2}=y+l_{e} \sin \varphi_{e} / 2$, $0^{\circ} \leq \varphi_{e} \leq 90^{\circ}$. The Gaussian dispersion from a line source or its equivalent finite line source (FLS) is illustrated in Figure 1. Further details can be found in Turner [35].

$$
\begin{aligned}
C_{e}(x, y) & =\int_{y_{e}^{1}}^{y_{e}^{2}} c(x, y, z=0) d y \\
& =\frac{q_{e}}{\pi \sigma_{y} \sigma_{z} u \sin \varphi_{e}} \int_{y_{e}^{1}}^{y_{e}^{2}} \exp \left(-\frac{y^{2}}{2 \sigma_{y}^{2}}\right) d y
\end{aligned}
$$

The emission concentration at any location $s$ with coordinate $\left(x_{s}, y_{s}\right)$ caused by a FLS $e \in F$ can be described using (16), where $F$ is the set that contains all the finite line sources of the network; $q_{e}$ is the emission concentration of FLS $e$; and obviously $q_{e}=q_{a}$ if $e \in F_{a}$, where $F_{a}$ is the set that contains all FLS of link $a$. Supposing that $p=y / \sigma_{y},(16)$ can be derived, where $\Phi(\bullet)$ is the cumulative probability density function of a standard normal distribution.

$$
\begin{aligned}
C_{e}\left(x_{s}, y_{s}\right) & =\frac{q_{e}}{\pi \sigma_{z} u \sin \varphi_{e}} \int_{y_{e}^{1} / \sigma_{y_{s}}}^{y_{e}^{2} / \sigma_{y_{s}}} \exp \left(-\frac{p^{2}}{2}\right) d p \\
& =\frac{\sqrt{2} q_{e}}{\sqrt{\pi} \sigma_{z} u \sin \varphi_{e}}\left[\Phi\left(\frac{y_{e}^{2}}{\sigma_{y_{s}}}\right)-\Phi\left(\frac{y_{e}^{1}}{\sigma_{y_{s}}}\right)\right]
\end{aligned}
$$

In this paper, we are particularly interested in modeling the emission exposure of transportation users. The emission exposure of a transportation user on link $a$ can be expressed as (17), which can be considered as the emission concentration multiplies the duration of exposure, where $t_{s}$ denotes 


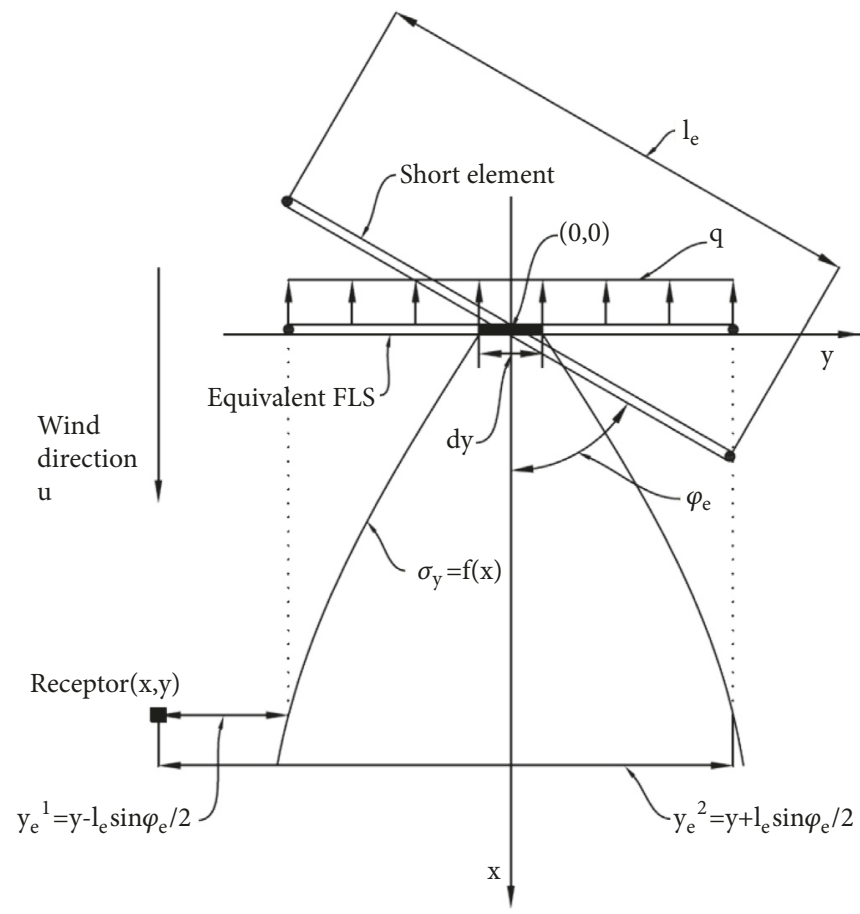

FIGURE 1: Dispersion from a short element/equivalent FLS.

the segment travel time of a finite line source $s \in F_{a}$, and $\sum_{s \in F_{a}} t_{s}=t_{a}$.

$$
\tau_{a}=\sum_{s \in F_{a}} \sum_{e \in F} C_{e}\left(x_{s}, y_{s}\right) t_{s}
$$

Assuming link $a$ is decomposed evenly and the link travel time is uniformly distributed along the link, then $t_{s}=t_{a} /\left|F_{a}\right|$, where $\left|F_{a}\right|$ represents the cardinality of set $F_{a}$. To make the cost function separable, we further assume that the emission concentration on link $a$ is only dependent upon the flow on this link. Therefore the emission exposure cost can be simplified as (18).

$$
\tilde{\tau}_{a}=\tilde{\tau}_{a}\left(v_{a}\right) \equiv \frac{t_{a}}{\left|F_{a}\right|} \sum_{s \in F_{a}} C_{s}\left(x_{s}, y_{s}\right)
$$

2.2. Multiple Equilibrium considering Human Exposure. In this section, we consider a generalized cost function for the transportation users, as in (19). The composite function comprises a travel time component $t_{a}$ and an emission exposure component $\widetilde{\tau}_{a}$; coefficient $\lambda$ is used to leverage the tradeoff between travel time and emission exposure.

$$
g_{a}=t_{a}+\lambda \widetilde{\tau}_{a}
$$

Therefore, the model for exposure of different players on the traffic emission can be formulated as below.

For the common UE player

$$
\min _{v^{U} \in \Omega^{U}} \sum_{a \in A} \int_{0}^{v_{a}^{U}} t_{a}\left(v_{a}^{E}+v_{a}^{K}+x\right) d x
$$

The function $t_{a}\left(v_{a}\right)$ is increasing, or $\partial t_{a}\left(v_{a}\right) / \partial v_{a}>$ $0, \forall a \in A$ and the function is convex $\partial^{2} t_{a}\left(v_{a}\right) / \partial t_{a}^{2} \geq 0, \forall a \epsilon$ $A$, so the minimization problem has a unique solution.

For the environmental advocacy UE player

$$
\min _{v^{E} \in \Omega^{E}} \sum_{a \in A} \int_{0}^{v_{a}^{E}}\left[t_{a}\left(v_{a}^{U}+v_{a}^{K}+x\right)+\lambda \widetilde{\tau}_{a}\left(v_{a}^{U}+v_{a}^{K}+x\right)\right] d x
$$

Note that the emission exposure cost $\widetilde{\tau}_{a}\left(v_{a}\right)$ is conditionally increasing and convex. In fact, it is easy to prove that the minimization problem has a unique solution under the condition of travel speed which is less than $150 \mathrm{~km} / \mathrm{h}$, which is true in most urban areas. The proof is provided in the appendix.

For the environmental advocacy $\mathrm{CN}$ players, $\forall k \in K$

$$
\begin{aligned}
& \min _{v^{k} \in \Omega^{k}} \sum_{a \in A}\left[t_{a}\left(v_{a}^{U}+v_{a}^{E}+v_{a}^{k}+v_{a}^{k-}\right)\right. \\
& \left.+\lambda \widetilde{\tau}_{a}\left(v_{a}^{U}+v_{a}^{E}+v_{a}^{k}+v_{a}^{k-}\right)\right] v_{a}^{k}
\end{aligned}
$$

Where the first derivative $t_{a}\left(v_{a}\right)+\lambda \widetilde{\tau}_{a}\left(v_{a}\right)+v_{a}^{k}\left(t_{a}^{\prime}\left(v_{a}\right)+\right.$ $\left.\lambda \widetilde{\tau}_{a}^{\prime}\left(v_{a}\right)\right)>0$ and the second derivative $2\left(t_{a}^{\prime}\left(v_{a}\right)+\lambda \widetilde{\tau}_{a}^{\prime}\left(v_{a}\right)\right)+$ $v_{a}^{k}\left(t_{a}^{\prime \prime}\left(v_{a}\right)+\lambda \widetilde{\tau}_{a}^{\prime \prime}\left(v_{a}\right)\right)>0, \forall a \in A$, so the minimization problem also has a unique solution.

Therefore, the minimization problems in (20), (21), and (22) can be characterized by a set of variational inequalities (VI):

$$
\sum_{a \in A} f_{a}^{\varphi}\left(v_{a}^{\varphi^{*}}, v_{a}^{-\varphi}\right)\left(v_{a}^{\varphi}-v_{a}^{\varphi^{*}}\right) \geq 0
$$

$$
\forall v^{\varphi} \in \Omega^{\varphi}, \forall \varphi \in \Phi
$$


TABLE 1: Network specifications.

\begin{tabular}{lccccccc}
\hline Link & $(1,3)$ & $(2,4)$ & $(1,5)$ & $(5,6)$ & $(2,5)$ & $(6,3)$ & $(6,4)$ \\
\hline$t_{a}^{0}(\mathrm{~min})$ & 3 & 3 & 1.5 & 1.5 & 1.5 & 1.5 \\
$c_{a}(\mathrm{veh} / \mathrm{hr})$ & 4000 & 4000 & 4000 & 8000 & 4000 & 4000 \\
$l_{a}(\mathrm{~km})$ & 4 & 4 & 2 & 2 & 2 & 4000 \\
\hline
\end{tabular}

Here $\Phi \equiv U \cup E \cup K, v_{a}^{-\varphi}$ is the total flow on link $a \in$ $A$ assigned by all players in $\Phi$ except $\varphi, \varphi \in \Phi$. Note that $f_{a}^{\varphi}\left(v_{a}^{\varphi}, v_{a}^{-\varphi}\right)$ is the perceived link cost of player $\varphi \in \Phi$, which is given as below:

$$
\begin{aligned}
& f_{a}^{\varphi}\left(v_{a}^{\varphi}, v_{a}^{-\varphi}\right) \\
& = \begin{cases}t_{a}\left(v_{a}^{-\varphi}+v_{a}^{\varphi}\right), & \text { if } \varphi \in U \\
g_{a}\left(v_{a}^{-\varphi}+v_{a}^{\varphi}\right), & \text { if } \varphi \in E \\
g_{a}\left(v_{a}^{-\varphi}+v_{a}^{\varphi}\right)+v_{a}^{\varphi} g_{a}^{\prime}\left(v_{a}^{-\varphi}+v_{a}^{\varphi}\right) & \text { if } \varphi=k, \forall k \in K\end{cases}
\end{aligned}
$$

Since the objective functions (20)-(22) are increasing and convex in most urban traffic settings, the VI problem (23) has a unique solution, and similar proof can be found in Yang et al. [9].

2.3. Solution Algorithm. In this paper, we applied a diagonalization method with nested method of successive averages (MSA) to solve the aforementioned VI problems. The solution approach is provided below.

Step 0. For iteration number $n=0$; use $t_{a}^{0}$ as the initial perceived link cost for all players and perform all-or-nothing assignment to get the initial total link flow $v_{a}^{(0)}$;

Step 1. Calculate the perceived cost of the common UE player, $f_{a}^{(n+1,1)}=f_{a}\left(v_{a}^{(n)}\right)$. Perform all-or-nothing assignment for the common UE player based on the perceived cost, and obtain a set of updated link flow $y_{a}^{(n+1,1)}$. Use step size $(\gamma)$; i.e., $\gamma=$ $1 / n$ to update the traffic flow on the network, $v_{a}^{(n+1,1)}=v_{a}^{(n)}+$ $\gamma\left(y_{a}^{(n+1,1)}-v_{a}^{(n)}\right)$

Step 2. Calculate the perceived link cost of the environmental advocacy UE player, $f_{a}^{(n+1,2)}=f_{a}\left(v_{a}^{(n+1,1)}\right)$. Perform all-ornothing assignment for the environmental UE player based on the perceived cost, and obtain a set of updated link flow $y_{a}^{(n+1,2)}$. Update the traffic flow on the network, $v_{a}^{(n+1,2)}=$ $v_{a}^{(n+1,1)}+\gamma\left(y_{a}^{(n+1,2)}-v_{a}^{(n+1,1)}\right)$

Step 3. Calculate the perceived link cost for each environmental advocacy CN player, $f_{a}^{(n+1, k+2)}=f_{a}\left(v_{a}^{(n+1, k+1)}\right), \forall k=$ $1,2,3 \cdots\|K\|$. Perform all-or-nothing assignment for each environmental advocacy CN player, and obtain a set of updated link flow $y_{a}^{(n+1, k+2)}$. Update the total traffic flow on the network, $v_{a}^{(n+1, k+2)}=v_{a}^{(n+1, k+1)}+\gamma\left(y_{a}^{(n+1, k+2)}-v_{a}^{(n+1, k+1)}\right)$;

Step 4 (convergence criteria). The convergence index is $\varepsilon_{\varphi}^{n}=$ $\sqrt{\sum_{a \in A}\left(v_{a}^{\varphi^{(n+1)}}-v_{a}^{\varphi^{(n)}}\right)^{2}} / \sum_{a \in A} v_{a}^{\varphi^{(n)}}, \varphi \in \Phi$. If $\varepsilon_{\varphi}^{n}$ satisfies

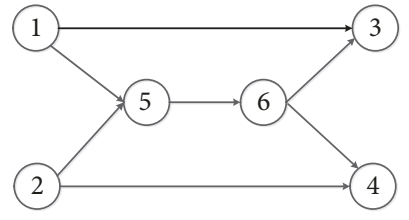

Figure 2: Study network.

the convergence condition, stop; otherwise, make $v_{a}^{(n+1)}=$ $v_{a}^{(n+1,\|K\|+2)}, n=n+1$, and proceed to step 1 .

\section{Numerical Experiment}

An idealistic six-node, seven-link transportation network, as shown in Figure 2, has been used to test the proposed modeling framework. There are two Origin-Destination (OD) pairs $(1,3)$ and $(2,4)$, both with the demand of 6000 vehicles per hour. The free speed of traffic flow is $80 \mathrm{~km} / \mathrm{h}$ for all links on the idealistic traffic network. The mean wind velocity is $0.5 \mathrm{~m} / \mathrm{s}$, and we use $\alpha=0.15, \beta=4$ to parameterize the BPR function. The network specifications and some model parameters are summarized in Table 1.

3.1. Environmental Advocacy UE (Case 1). In the first case, we assume that all transportation users follow the first Wardropian principle and all the users take the same composite cost function. There are two paths (e.g., path 1-3 and path 1-5-6-3) for OD pair $(1,3)$.

Figure 3 depicts the change of traffic flow, travel time, and emission exposure on the two paths with respect to the change of parameter $\lambda$. It is found that when $\lambda=0$, the travel time costs of the two paths are identical, which satisfy the UE principle. It is found that when $\lambda$ increases, people become more concerned about their emission exposure, as a result, some flow has switched from path 1-3 to path 1-5-6-3 and the emission exposure cost as well as the travel time on path 1-3 decreased. Noteworthy the travel time on path 1-5-6-3 only slightly increases as the capacity on this path is much larger than the flow, therefore the marginal effects of flow is quite small. The results for path 2-4 and 2-5-6-4 are not shown here since the network and OD volumes are symmetric. The results show that the route choice patterns have been changed by considering the emission exposure cost in the cost function.

Figure 4 shows the system total cost (represented by $\left.\sum_{a}\left[t_{a}\left(v_{a}\right)+\tilde{\tau}_{a}\left(v_{a}\right)\right]\right)$, travel time, and emission exposure with respect to the change of $\lambda$. Per the specific network setting, we find the total cost decreased as $\lambda$ increasing. This suggests that as travelers become more concerned about their exposure to vehicular emissions, the system emission exposure, travel 


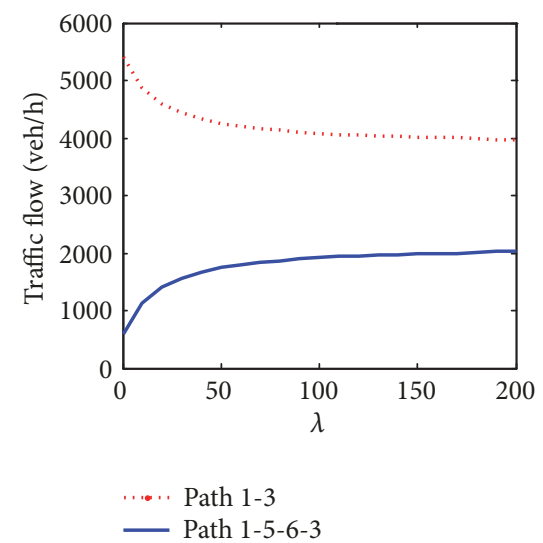

(a)

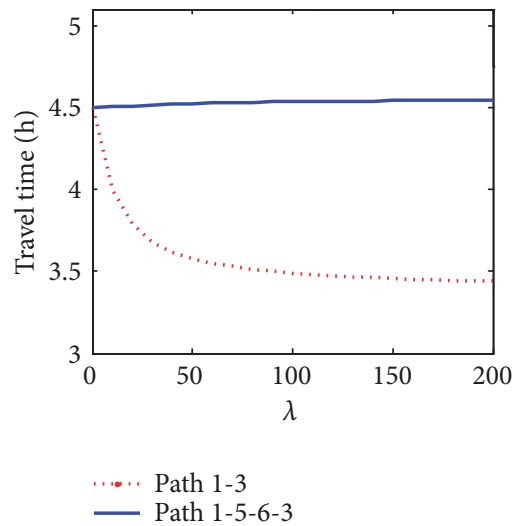

(b)

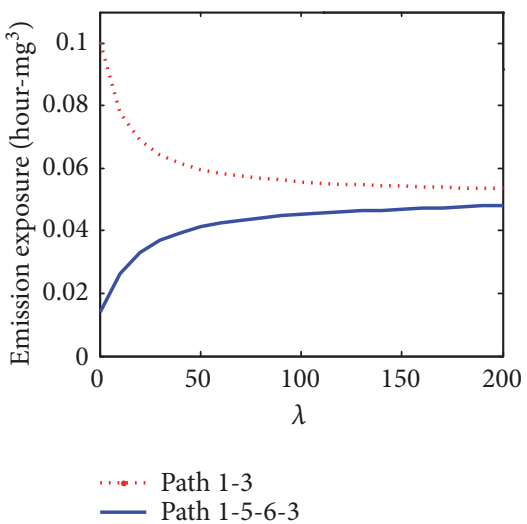

(c)

FiguRE 3: Path-based results of the environmental advocacy UE: (a) traffic flow; (b) travel time; (c) emission exposure.

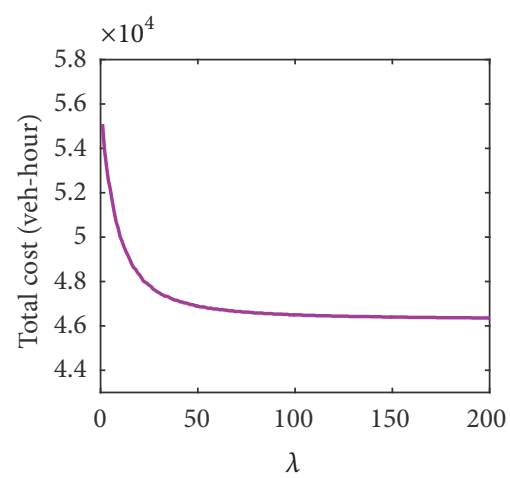

(a) $\sum_{a}\left[t_{a}\left(v_{a}\right)+\tilde{\tau}_{a}\left(v_{a}\right)\right]$

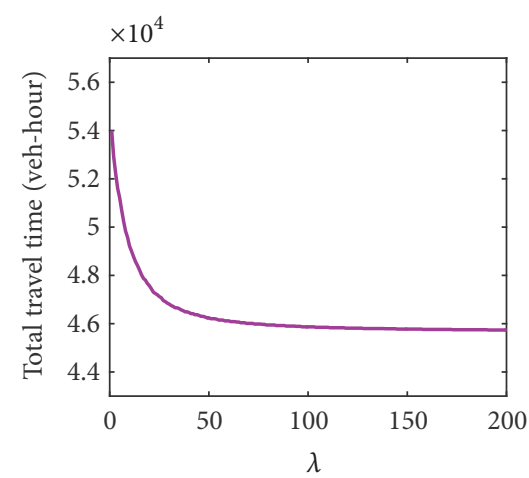

(b) $\sum_{a} t_{a}\left(v_{a}\right)$

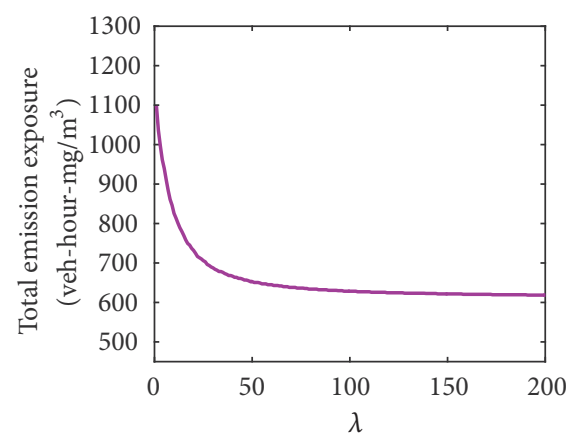

(c) $\sum_{a} \tilde{\tau}_{a}\left(v_{a}\right)$

Figure 4: System costs of case 1: (a) total cost; (b) travel time; (c) emission exposure.

time, and the total cost all get reduced, i.e., Pareto improving solutions are achieved.

3.2. Multiple Equilibrium with a Common UE and an Environmental Advocacy UE (Case 2). In the second case, we assume there are a common UE player and an environmental advocacy UE player. We have only two types of players in the case; therefore the proposed approach simplified can be to tackle this case. By setting different user proportions, we observe the change of system total cost, travel time, and emission exposure with respect to $\lambda$; the results are provided in Figure 5. Here $\theta$ represents the percentage of the environmental advocacy UE users, and $(1-\theta)$ represents the percentage of the common UE users.

When $\theta=0$, since only the common UE users exist, the system total cost, travel time, and emission exposure remain unchanged with the change of $\lambda$. In Figure 5(a), the total cost first reduces when a small percentage of users become environmental advocacy UE users. The cost remains stable when the percentage of environmental advocacy UE users becomes larger than $20 \%$. Similar trends are discovered for the system travel time and emission exposure. This suggests that the system gets better if more users become environmental advocators. In an operation perspective, the change of a small percentage of users should already lead to good system improvement.

3.3. Multiple Equilibrium with a Common UE and an Environmental Advocacy $\mathrm{CN}$ (Case 3). We further look at the scenario with a common UE player and an environmental advocacy CN player on the network. This scenario is practically meaningful since there may be some environmentalcost based routing models (e.g., smartphone apps) that provide routing services to minimize individual/group-wide emission exposure.

The results of this case are in general similar to case 2 . As shown in Figure 6(a), as $\theta$ increasing, the total cost first decreases and becomes stable when $\theta$ becomes larger than $60 \%$. Figures $6(\mathrm{~b})$ and $6(\mathrm{c})$ reveal similar trends for the travel time and emission exposure cost.

The results from case 3 are compared to the ones in case 2, as indicated in Figure 7. Figure 7(a) shows that when 50\% users are environmental advocacy users, the total cost of case 2 is larger than case 3 ; when $\lambda<50$, the result is consistent with common sense that cooperative behavior leads to reduced system cost. But as the users seriously concern their 


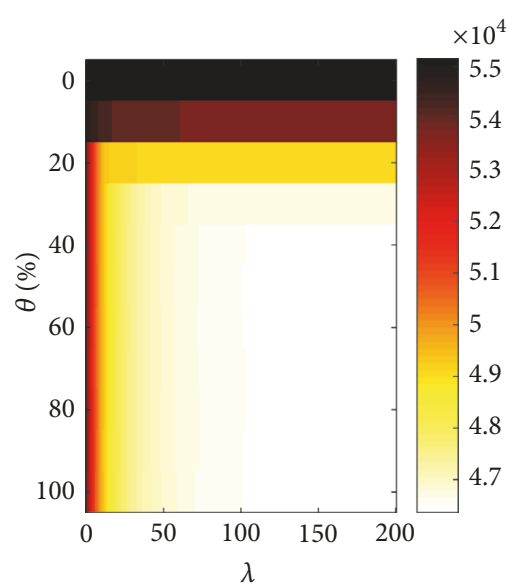

(a) $\sum_{a}\left[t_{a}\left(v_{a}\right)+\tilde{\tau}_{a}\left(v_{a}\right)\right]$

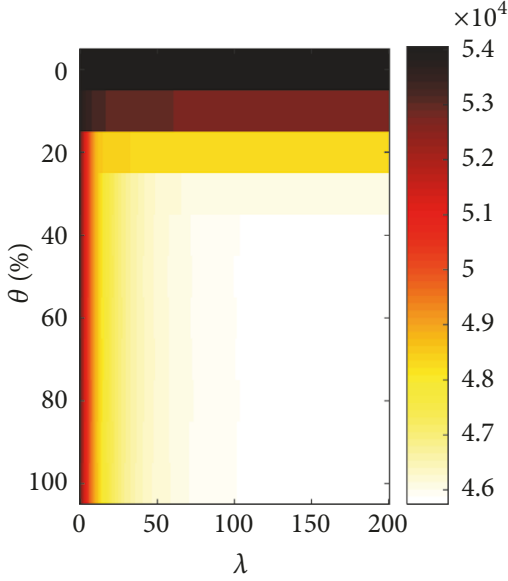

(b) $\sum_{a} t_{a}\left(v_{a}\right)$

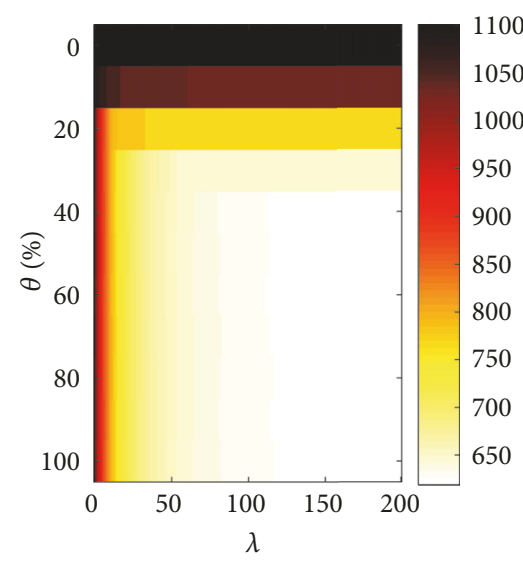

(c) $\sum_{a} \tilde{\tau}_{a}\left(v_{a}\right)$

FIGURE 5: System costs with a common UE and an environmental advocacy UE: (a) total cost; (b) travel time; (c) emission exposure.

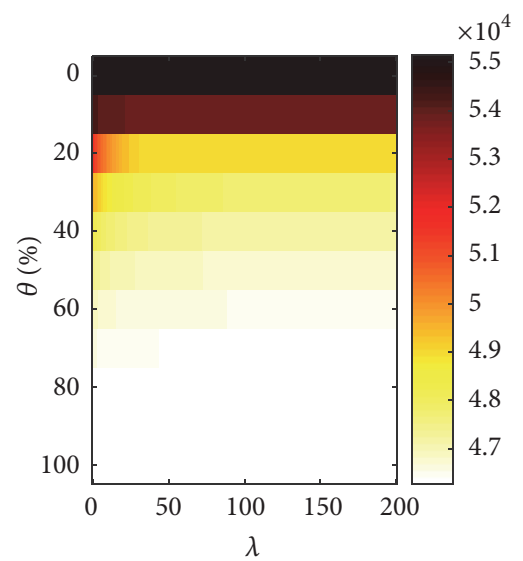

(a) $\sum_{a}\left[t_{a}\left(v_{a}\right)+\tilde{\tau}_{a}\left(v_{a}\right)\right]$

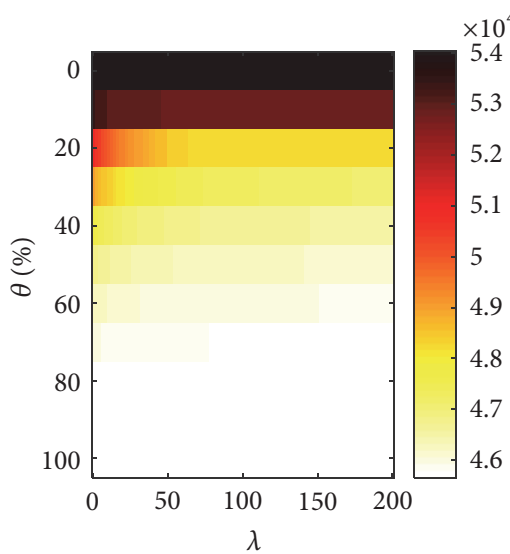

(b) $\sum_{a} t_{a}\left(v_{a}\right)$

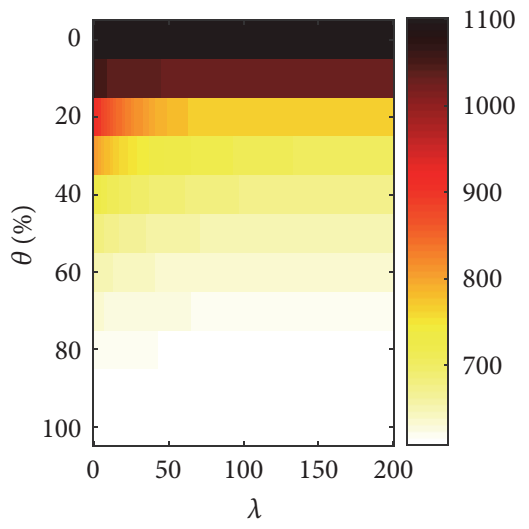

(c) $\sum_{a} \tilde{\tau}_{a}\left(v_{a}\right)$

FIGURE 6: System costs with a common UE and an environmental advocacy CN; (a) total cost; (b) travel time; (c) emission exposure.

emission exposure (e.g., $\lambda \geq 50$ ), the cooperative behavior $(\mathrm{CN})$ results in worse system performance. Figure 7(b) shows that when $70 \%$ or more users are environmental advocacy users, the cooperative behavior $(\mathrm{CN})$ is always better than the competitive behavior, and such effect is more significant at lower $\lambda$ value.

\section{Concluding Remarks}

In this study, an integrated modeling framework was applied to analytically estimate vehicular emission and concentration within the traffic network. The framework was used to calculate travelers' exposure to emissions, which was considered as a part of the generalized travel cost. The experimental results showed that the route choice behaviors have been changed by considering the emission exposure cost in the cost function. Per the specific network setting, we found that as travelers become more concerned about their exposure to vehicular emissions, the system emission exposure, travel time, and the total cost all get reduced; i.e., Pareto improving solutions are achieved. By analyzing the multiple equilibrium behaviors, we found the system gets better if more users become environmental advocates. For example, in the case that all users follow common UE principle, when $20 \%$ of users become environmental advocates, the total system cost reduces about $11 \%$. And the change of a small percentage of users should already lead to good system improvement. By comparing the case with a common UE and an environmental advocacy UE to the case with a common UE and an environmental advocacy $\mathrm{CN}$, we found that cooperative behaviors $(\mathrm{CN})$ lead to better system performance when people's concerns of emission exposure remain at a rational level. The findings of the paper clearly show the necessity and importance of providing exposure-based routing applications to reduce human exposures and to improve the overall performance of transportation networks. As travelers are becoming more concerned of their emission exposure, such eco-routing models are more behavioral sound compared to the conventional emission-based routing models. 


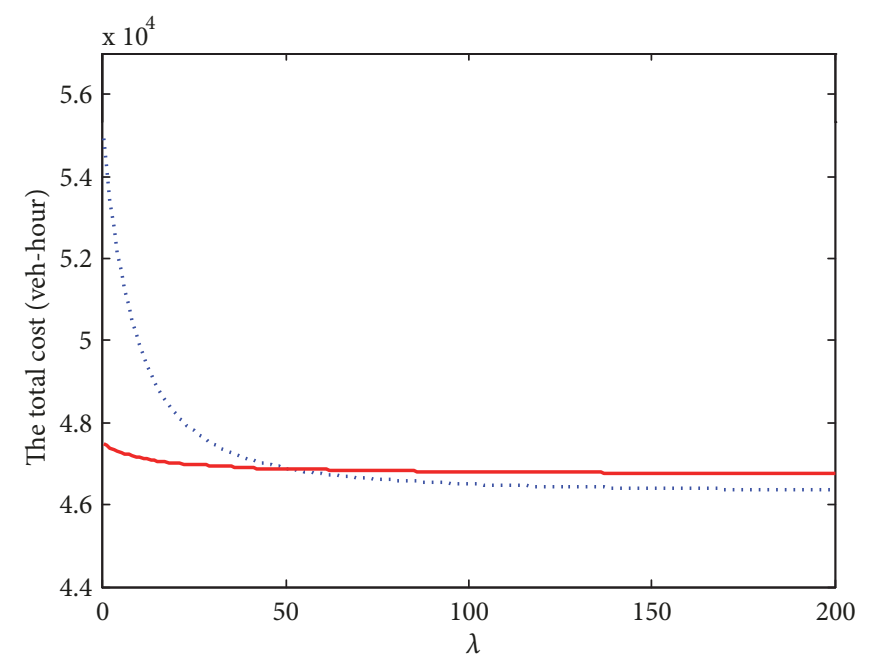

..... The environmental advocacy UE player with common UE player The environmental advocacy CN player with common UE player

(a)

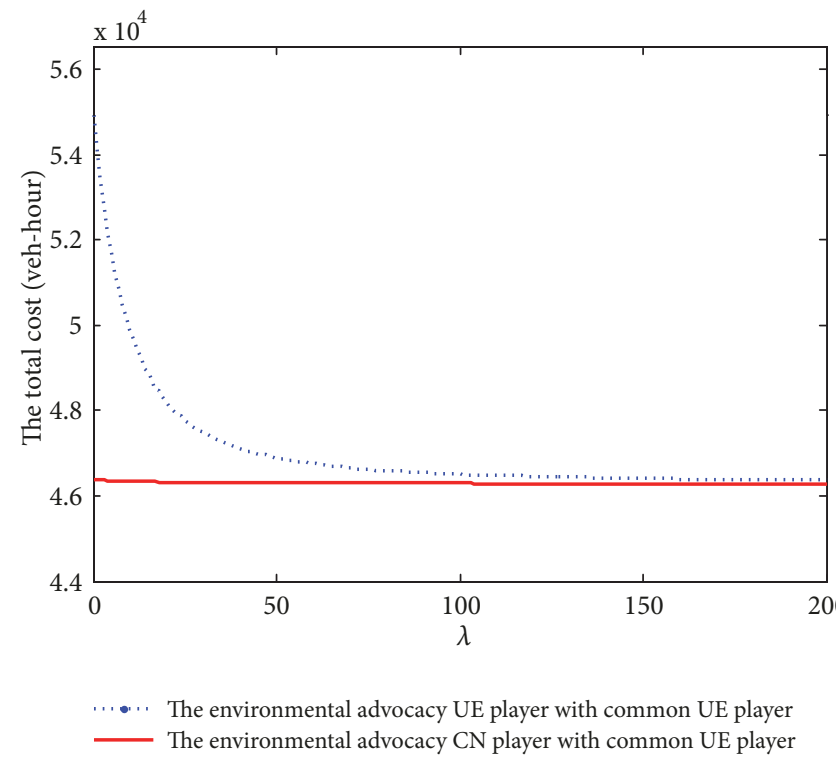

(b)

Figure 7: Comparison of case 2 and case 3 in total cost $\left(\left(\sum_{a}\left[t_{a}\left(v_{a}\right)+\widetilde{\tau}_{a}\left(v_{a}\right)\right]\right)\right)$ : (a) total cost when $\theta=0.5$ and (b) total cost when $\theta=0.7$.

It is worthy to mention that human exposure to vehicular emissions is a very complex problem. This article only provided an integrated approach to capture human exposure in route choice. Many modeling details need to be further studied. In the future, the authors will test the proposed framework using more realistic traffic flow and dispersion models and using real traffic networks. Another possible extension is to consider dynamic and elastic demand in the proposed multiple equilibrium. More results and findings will be provided in our subsequent work.

\section{Appendix}

$$
\begin{aligned}
& \tilde{\tau}_{a}\left(v_{a}\right)=\frac{t_{a}}{\left|F_{a}\right|} \sum_{s \in F_{a}} C_{s}\left(x_{s}, y_{s}\right)=\frac{t_{a}}{\left|F_{a}\right|} \\
& \cdot \sum_{s \in F_{a}} \frac{\sqrt{2}}{\sqrt{\pi} \sigma_{z} u \sin \varphi_{s}}\left(\Phi\left(\frac{y_{s}^{2}}{\sigma_{y}}\right)-\Phi\left(\frac{y_{s}^{1}}{\sigma_{y}}\right)\right) q_{s} \\
& =0.2038 \frac{t_{a}}{\left|F_{a}\right|} \\
& \cdot \sum_{s \in F_{a}} \frac{\sqrt{2}}{\sqrt{\pi} \sigma_{z} u \sin \varphi_{s}}\left(\Phi\left(\frac{y_{s}^{2}}{\sigma_{y}}\right)-\Phi\left(\frac{y_{s}^{1}}{\sigma_{y}}\right)\right) \\
& \cdot \frac{v_{a} t_{a}}{3600 l_{a}} e^{0.7962 l_{a} / t_{a}}=\frac{0.2038}{3600 l_{a}\left|F_{a}\right|} \\
& \cdot \sum_{s \in F_{a}} \frac{\sqrt{2}}{\sqrt{\pi} \sigma_{z} u \sin \varphi_{s}}\left(\Phi\left(\frac{y_{s}^{2}}{\sigma_{y}}\right)-\Phi\left(\frac{y_{s}^{1}}{\sigma_{y}}\right)\right) \\
& \cdot v_{a} t_{a}^{2} e^{0.7962 l_{a} / t_{a}}=\operatorname{const} * v_{a} t_{a}^{2} e^{0.7962 l_{a} / t_{a}}
\end{aligned}
$$

Therefore the first derivative of $\widetilde{\tau}_{a}$ with respect to $v_{a}$ can be described as in

$$
\begin{aligned}
& \tilde{\tau}_{a}^{\prime}\left(v_{a}\right)=\frac{d \widetilde{\tau}_{a}}{d v_{a}} \\
& =\text { const } \\
& \quad * e^{0.7962 l_{a} / t_{a}}\left(t_{a}^{2}+\frac{d t_{a}}{d v_{a}}\left(2 v_{a} t_{a}-0.7962 l_{a} v_{a}\right)\right)
\end{aligned}
$$

Since $t_{a}^{2} \geq 0$ and $d t_{a} / d v_{a} \geq 0$, we have $\tilde{\tau}_{a}^{\prime}\left(v_{a}\right) \geq 0$ if $l_{a} / t_{a} \leq(2 * 60) / 0.7962=150.7 \mathrm{~km} / \mathrm{h}$. That is, $\widetilde{\tau}_{a}\left(v_{a}\right)$ is an increasing function if link speed is less than $150.7 \mathrm{~km} / \mathrm{h}$.

The second derivative of $\widetilde{\tau}_{a}$ with respect to $v_{a}$ can be calculated as in

$$
\begin{aligned}
& \tilde{\tau}_{a}^{\prime \prime}\left(v_{a}\right)=\frac{d \tilde{\tau}_{a}^{2}}{d v_{a}{ }^{2}}=\text { const } * e^{0.7962 l_{a} / t_{a}}\left(t_{a}{ }^{2}\right. \\
& \left.+\frac{d t_{a}}{d v_{a}}\left(2 v_{a} t_{a}-0.7962 l_{a} v_{a}\right)\right)=\text { const } \\
& * e^{0.7962 l_{a} / t_{a}}\left(2 t_{a} \frac{d t_{a}}{d v_{a}}+\frac{d t_{a}}{d v_{a}}\left(2 t_{a}-0.7962 l_{a}\right)\right. \\
& \left.+2 v_{a}\left(\frac{d t_{a}}{d v_{a}}\right)^{2}+\frac{d t_{a}^{2}}{d v_{a}^{2}}\left(2 v_{a} t_{a}-0.7962 l_{a} v_{a}\right)\right) \\
& -\operatorname{const} * e^{0.7962 l_{a} / t_{a}} \frac{0.7962 l_{a}}{t_{a}^{2}} \frac{d t_{a}}{d v_{a}}\left(t_{a}{ }^{2}\right. \\
& \left.+\frac{d t_{a}}{d v_{a}}\left(2 v_{a} t_{a}-0.7962 l_{a} v_{a}\right)\right)=\text { const } \\
& * 2 e^{0.7962 l_{a} / t_{a}} \frac{d t_{a}}{d v_{a}}\left(2 t_{a}-0.7962 l_{a}\right)+\text { const }
\end{aligned}
$$




$$
\begin{aligned}
& * e^{0.7962 l_{a} / t_{a}} \frac{d t^{2}}{d v_{a}{ }^{2}}\left(2 v_{a} t_{a}-0.7962 l_{a} v_{a}\right)+\text { const } \\
& * e^{0.7962 l_{a} / t_{a}} v_{a}\left(\frac{d t_{a}}{d v_{a}}\right)^{2}\left(1+\left(1-\frac{0.7962 l_{a}}{t_{a}}\right)^{2}\right)
\end{aligned}
$$

Similarly, we have $\widetilde{\tau}^{\prime \prime}{ }_{a}\left(v_{a}\right)>0$ if $l_{a} / t_{a} \leq(2 * 60) / 0.7962=$ $150.7 \mathrm{~km} / \mathrm{h}$. Therefore $\widetilde{\tau}_{a}\left(v_{a}\right)$ is strictly convex if link speed is less than $150.7 \mathrm{~km} / \mathrm{h}$. This completes the proof.

\section{Notations}

$N$ : The set of nodes of the network

A: The set of links of the network

$U:$ The common UE player on the network

$E$ : The environmental advocacy UE player on the network

$K$ : The set of environmental advocacy $\mathrm{CN}$ players on the network, $k \in K$

$W^{U}$ : The set of OD pairs of pertaining to the common UE player

$W^{E}$ : The set of OD pairs pertaining to the environmental advocacy UE player

$W^{k}$ : The set of OD pairs pertaining to the environmental advocacy $\mathrm{CN}$ player(s)

$W: \quad \mathrm{W} \equiv W^{U} \cup W^{E} \cup_{k \in K} W^{k}$

$D_{w}^{U}$ : The demand between OD pair $w \in W^{U}$

$D_{w}^{E}$ : The demand between OD pair $w \in W^{E}$

$D_{w}^{k}$ : The demand between OD pair $w \in W^{k}, k \in K$

$P_{w}$ : The set of paths between OD pair $w \in W$

$P^{U}: P^{U} \equiv \cup_{w \in W^{U}} P_{w}$

$P^{E}: \quad P^{E} \equiv \cup_{w \in W^{E}} P_{w}$

$P^{K}: \quad P^{K} \equiv \cup_{w \in W^{k}, k \in K} P_{w}$

$P: \quad P \equiv P^{U} \cup P^{E} \cup P^{K}$

$f_{p}^{U}$ : The flow on path $p$ pertaining to the common UE player

$f_{p}^{E}$ : The flow on path $p$ pertaining to the environmental advocacy UE player

$f_{p}^{k}$ : The flow on path $p$ pertaining to the environmental advocacy $\mathrm{CN}$ player $k$

$\delta_{a p}: 1$ if link $a \in A$ is on route $p \in P$ and 0 otherwise

$v_{a}^{U}$ : The flow of link $a$ that follows the common UE principle

$v_{a}^{E}$ : The flow of link $a$ controlled by the environmental advocacy UE player

$v_{a}^{k}$ : The flow of link $a$ controlled by environmental advocacy $\mathrm{CN}$ player $k$

$v_{a}^{K}: \quad v_{a}^{K} \equiv \sum_{k \in K} v_{a}^{k}$

$v_{a}: \quad$ Total flow on link $a, v_{a} \equiv v_{a}^{U}+v_{a}^{E}+v_{a}^{K}$

$v^{U}: \quad v^{U} \equiv\left(\cdots, v_{a-1}^{U}, v_{a}^{U}, v_{a+1}^{U}, \cdots\right)$

$v^{E}: \quad v^{E} \equiv\left(\cdots, v_{a-1}^{E}, v_{a}^{E}, v_{a+1}^{E}, \cdots\right)$

$v^{k}: \quad v^{k} \equiv\left(\cdots, v_{a-1}^{k}, v_{a}^{k}, v_{a+1}^{k}, \cdots\right)$

$v^{K}: \quad v^{K} \equiv\left(\cdots, v^{k-1}, v^{k}, v^{k+1}, \cdots\right)$

$\tau_{a}$ : Emission exposure on link $a$

$\tilde{\tau}_{a}:$ Simplified emission exposure on link $a$ $t_{a}\left(v_{a}\right)$ : Travel time on link $a$

$e_{a}\left(v_{a}\right)$ : CO emission rate on link $a$

$q_{a}\left(v_{a}\right)$ : CO emission source strength on link $a$

$F$ : $\quad$ The set that contains all finite line sources of the network

$F_{a}: \quad$ The set that contains all finite line sources of link $a$

$g_{a}: \quad$ A generalized cost function of link $a$.

\section{Data Availability}

The data used to support the findings of this study are available from the corresponding author upon request.

\section{Conflicts of Interest}

The authors declare that they have no conflicts of interest.

\section{Acknowledgments}

The paper was improved and extended based on a conference version presented at CICTP 2018. Information of the conference paper can be found at http://www.cota-home.org/ Docs/Conference/CICTP2018/TechSession.pdf. The authors are supported by the National Natural Science Foundation of China (NSFC) via grant 71701173. Any opinions, findings, and conclusions or recommendations expressed in this paper are those of the authors and do not necessarily reflect the views of the NSFC.

\section{References}

[1] US Environmental Protection Agency (EPA). (2017). Air Pollutant Emissions Trends Data, Retrieved (May 23, 2018) from https://www.epa.gov/transportation-air-pollution-and-climatechange/accomplishments-and-success-air-pollution-transportation.

[2] X. Tian, H. Dai, Y. Geng et al., "Economic impacts from PM2.5 pollution-related health effects in China's road transport sector: A provincial-level analysis," Environment International, vol. 115, pp. 220-229, 2018.

[3] US Environmental Protection Agency (EPA). (2011). Nationalscale air toxics assessment. Retrieved (July 23, 2016) from https:/www.epa.gov/national-air-toxics-assessment/2011-nationalair-toxics-assessment.

[4] Y. Yin and S. Lawphongpanich, "Internalizing emission externality on road networks," Transportation Research Part D: Transport and Environment, vol. 11, no. 4, pp. 292-301, 2006.

[5] A. Skabardonis, N. Geroliminis, and E. Christofa, "Vehicle emissions estimation under oversaturated conditions along signalized arterials," in Proceedings of the 91th TRB Annual Meeting (CD), Washington, DC, USA, 2012.

[6] Z. Sun, P. Hao, X. Ban, and D. Yang, "Trajectory-based vehicle energy/emissions estimation for signalized arterials using mobile sensing data," Transportation Research Part D: Transport and Environment, vol. 34, pp. 27-40, 2015.

[7] W. Zhu, S. Ma, and J. Tian, "Optimizing congestion and emissions via tradable credit charge and reward scheme without initial credit allocations," Physica A: Statistical Mechanics and its Applications, vol. 465, pp. 438-448, 2017. 
[8] P. T. Harker, "Multiple equilibrium behaviors on networks," Operations Research Society of America. Transportation Science Section. Transportation Science, vol. 22, no. 1, pp. 39-46, 1988.

[9] H. Yang, X. Zhang, and Q. Meng, "Stackelberg games and multiple equilibrium behaviors on networks," Transportation Research Part B: Methodological, vol. 41, no. 8, pp. 841-861, 2007.

[10] X. Zhang, H. Yang, and H. Huang, "Multiclass multicriteria mixed equilibrium on networks and uniform link tolls for system optimum," European Journal of Operational Research, vol. 189, no. 1, pp. 146-158, 2008.

[11] M. Meng, C. F. Shao, Y. D. Wong, and J. Zhang, "Multimodal Network Equilibrium with Stochastic Travel Times," Mathematical Problems in Engineering, vol. 2014, Article ID 136872, 8 pages, 2014.

[12] X. Yang, X. J. Ban, and R. Ma, "Mixed equilibria with common constraints on transportation networks," Networks and Spatial Economics, vol. 17, no. 2, pp. 547-579, 2017.

[13] F. He, Y. Yin, N. Shirmohammadi, and Y. M. Nie, "Tradable credit schemes on networks with mixed equilibrium behaviors," Transportation Research Part B: Methodological, vol. 57, pp. 4765, 2013.

[14] A. Alzuhairi, M. Aldhaheri, Z.-B. Sun, J.-S. Oh, and V. Kwigizile, "Vehicular emissions and concentrations in school zones: A case study," Journal of Central South University, vol. 23, no. 7, pp. 1778-1785, 2016.

[15] M. Barth, G. Scora, and T. Younglove, "Estimating emissions and fuel consumption for different levels of freeway congestion," Transportation Research Record, no. 1664, pp. 47-57, 1999.

[16] G. Scora and M. Barth, Comprehensive Modal Emissions Model (CMEM), version 3.1, User's Guide. 2006. LIST OF TABLES TABLE, 1, 2014.

[17] H. Rakha, K. Ahn, and A. Trani, "Development of VT-Micro model for estimating hot stabilized light duty vehicle and truck emissions," Transportation Research Part D: Transport and Environment, vol. 9, no. 1, pp. 49-74, 2004.

[18] D. Llopis-Castelló, A. M. Pérez-Zuriaga, F. J. CamachoTorregrosa, and A. García, "Impact of horizontal geometric design of two-lane rural roads on vehicle co2 emissions," Transportation Research Part D: Transport and Environment, vol. 59, pp. 46-57, 2018.

[19] US Environmental Protection Agency (EPA), (2014). MOVES2014 a user guide, Retrieved (July 23, 2016) from https:// www3.epa.gov/otaq/models/moves/documents/420b15095.pdf.

[20] C. Wallace, K. Courage, M. Hadi, and A. Gan, TRANSYT-7F User's Guide, University of Florida, Gainesville, FL, USA, 1998.

[21] K. Boriboonsomsin, M. J. Barth, W. Zhu, and A. Vu, "Ecorouting navigation system based on multisource historical and real-time traffic information," IEEE Transactions on Intelligent Transportation Systems, vol. 13, no. 4, pp. 1694-1704, 2012.

[22] A. Skabardonis, N. Geroliminis, and E. Christofa, "Prediction of vehicle activity for emissions estimation under oversaturated conditions along signalized arterials," Journal of Intelligent Transportation Systems: Technology, Planning, and Operations, vol. 17, no. 3, pp. 191-199, 2013.

[23] R. Ma, X. Ban, and W. Y. Szeto, "Emission modeling and pricing in dynamic traffic networks," Transportation Research Procedia, vol. 9, pp. 106-129, 2015.

[24] R. Saedi, R. Verma, A. Zockaie, M. Ghamami, and T. Gates, "A Framework for Incorporating the Network-Wide Fundamental Diagram into Large-Scale Emission Estimation," Journal of Transport Health, vol. 9, pp. S54-S55, 2018.
[25] M. R. Beychok, Fundamentals of Stack Gas Dispersion, Newport Beach, CA, USA, 1994.

[26] S. P. Arya, Air Pollution Meteorology and Dispersion, Oxford University Press, New York, NY, USA, 1999.

[27] T. Chai, R. Draxler, and A. Stein, "Source term estimation using air concentration measurements and a Lagrangian dispersion model - Experiments with pseudo and real cesium-137 observations from the Fukushima nuclear accident," Atmospheric Environment, vol. 106, pp. 241-251, 2015.

[28] N. Sharma, K. K. Chaudhry, and C. V. Chalapati Rao, "Vehicular pollution prediction modelling: A review of highway dispersion models," Transport Reviews, vol. 24, no. 4, pp. 409-435, 2004.

[29] P. E. Benson, "CALINE4 - A dispersion model for predicting air pollutant concentrations near roadways," Report No. FHWA/CA/TL-84-15, California Department of Transportation, Sacramento, CA, USA, 1984.

[30] P. Benson, CALINE3 line source dispersion model, FHWACA-TL-84-15, California Department of Transportation, Sacramento, CA, USA, 1984.

[31] J. Levitin, J. Härkönen, J. Kukkonen, and J. Nikmo, "Evaluation of the CALINE4 and CAR-FMI models against measurements near a major road," Atmospheric Environment, vol. 39, no. 25, pp. 4439-4452, 2005.

[32] S. Samaranayake, S. Glaser, D. Holstius et al., "Real-Time Estimation of Pollution Emissions and Dispersion from Highway Traffic," Computer-Aided Civil and Infrastructure Engineering, vol. 29, no. 7, pp. 546-558, 2014.

[33] T. L. Alderete, R. B. Jones, Z. Chen et al., "Exposure to trafficrelated air pollution and the composition of the gut microbiota in overweight and obese adolescents," Environmental research, vol. 161, pp. 472-478, 2018.

[34] H. Yang and X. Zhang, "Existence of anonymous link tolls for system optimum on networks with mixed equilibrium behaviors," Transportation Research Part B: Methodological, vol. 42, no. 2, pp. 99-112, 2008.

[35] D. B. Turner, Workbook of atmospheric dispersion estimates: an introduction to dispersion modeling, CRC Press, London, UK, 2nd edition, 1994.

[36] X. Liu, A. Godbole, C. Lu, G. Michal, and P. Venton, "Optimisation of dispersion parameters of Gaussian plume model for $\mathrm{CO}_{2}$ dispersion," Environmental Science and Pollution Research, vol. 22, no. 22, pp. 18288-18299, 2015. 


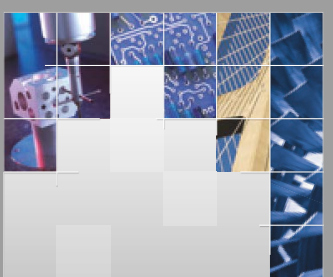

\section{Enfincering}
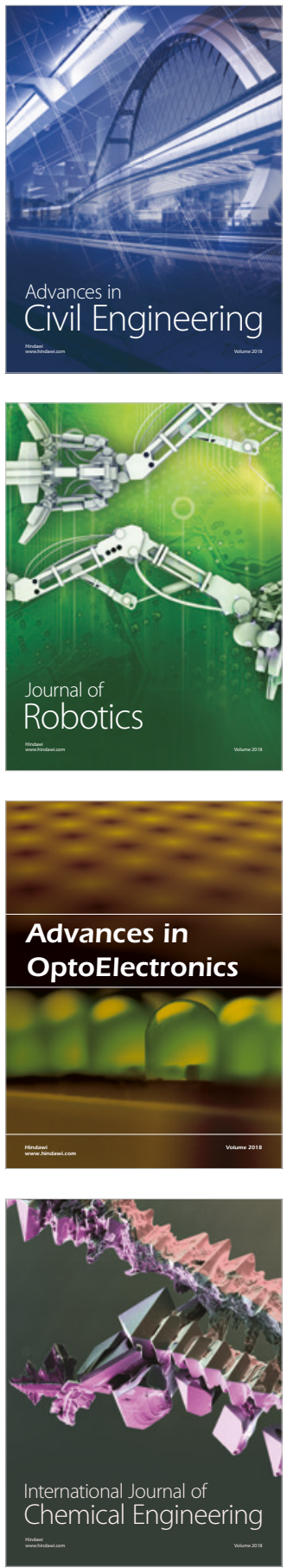

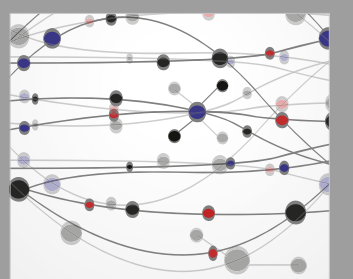

\section{Rotating \\ Machinery}

The Scientific World Journal

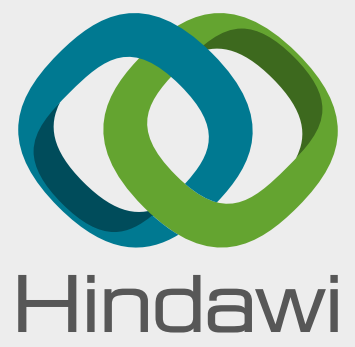

Submit your manuscripts at

www.hindawi.com
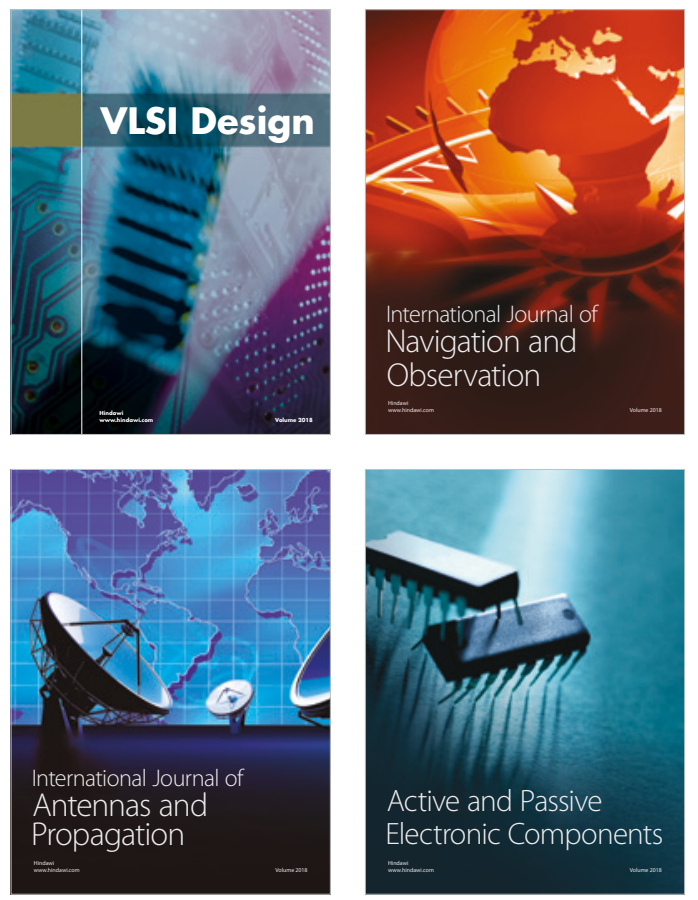
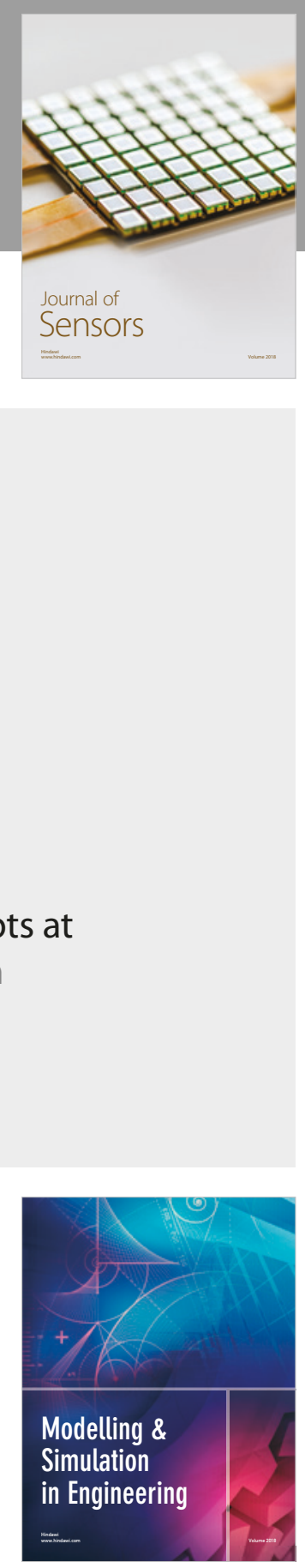

\section{Advances \\ Multimedia}
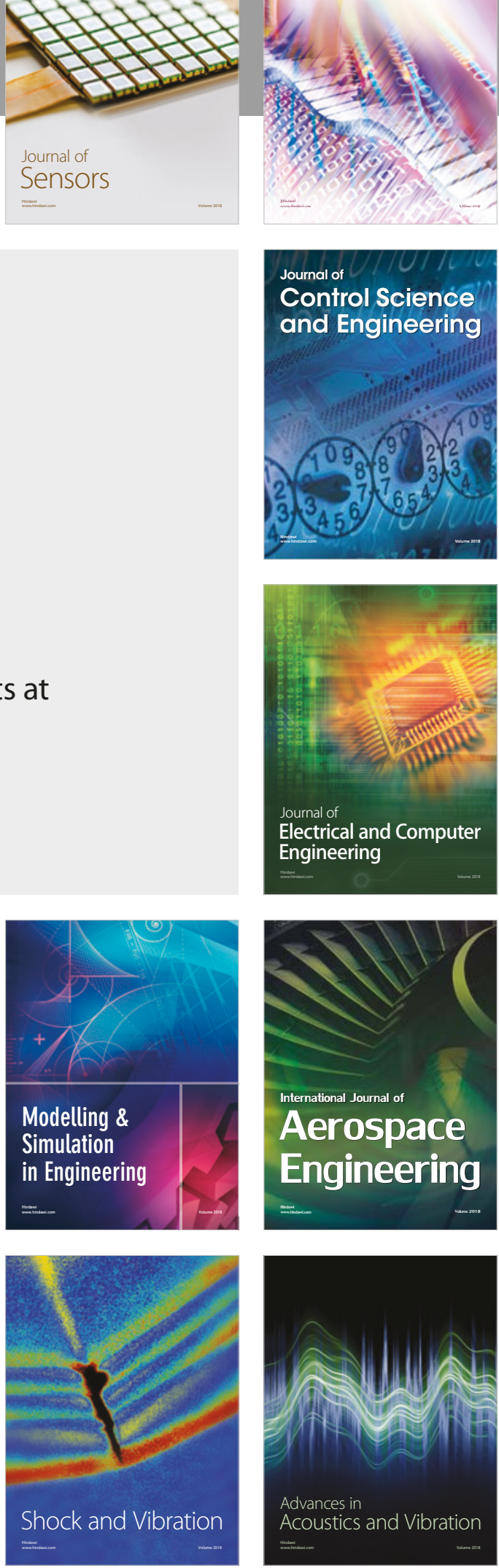